\title{
Bargmann invariants and off-diagonal geometric phases for multilevel quantum systems: A unitary-group approach
}

\author{
N. Mukunda* \\ Centre for Theoretical Studies, Indian Institute of Science, Bangalore 560012, India \\ Arvind $^{\dagger}$ \\ Department of Physics, Guru Nanak Dev University, Amritsar 143005, India \\ S. Chaturvedi ${ }^{\ddagger}$ \\ Department of Physics, University of Hyderabad, Hyderabad 500046, India \\ R. Simon ${ }^{\S}$ \\ Institute of Mathematical Sciences, CIT Campus, Chennai 600113, India
}

\begin{abstract}
We investigate the geometric phases and the Bargmann invariants associated with multilevel quantum systems. In particular, we show that a full set of "gauge-invariant" objects for an $n$-level system consists of $n$ geometric phases and $\frac{1}{2}(n-1)(n-2)$ algebraically independent four-vertex Bargmann invariants. In the process of establishing this result, we develop a canonical form for $\mathrm{U}(n)$ matrices that is useful in its own right. We show that the recently discovered "off-diagonal" geometric phases [N. Manini and F. Pistolesi, Phys. Rev. Lett. 8, 3067 (2000)] can be completely analyzed in terms of the basic building blocks developed in this work. This result liberates the off-diagonal phases from the assumption of adiabaticity used in arriving at them.
\end{abstract}

\section{INTRODUCTION}

The notion of geometric phase, though defined originally in the context of adiabatic, unitary, and cyclic evolution [1], has now come to be recognized as a direct consequence of the geometry of the complex Hilbert space and that of the associated ray space [2]. The quantum kinematic [2] picture, which has thus emerged, provides a much wider setting for the notion of the geometric phase by rendering superfluous the various assumptions that attended its original discovery [1] and subsequent development [3-5]. In particular, the requirement of cyclic evolution is no longer necessary and it becomes possible to ascribe a geometric phase to any open curve in the Hilbert space that has nonorthogonal unit vectors as its end points. Further, following the quantum kinematic approach [6], one is led in a natural way to the intimate relationship that exists between the geometric phase and the $n$-vertex Bargmann invariants [7] and that between the geometric phase and Hamilton's theory of turns [8]. As an application of this approach, the Gouy phase (the phase jump experienced by a focused light beam as it crosses the focus), discovered over a hundred years ago, has been shown to be a four-vertex Bargmann invariant [9].

In the present work, we develop the quantum kinematic approach for the special case of unitary evolution of an $n$-level system. It turns out that, in the present context, it becomes necessary to introduce Bargmann invariants con-

\footnotetext{
*Email address: nmukunda@cts.iisc.ernet.in

${ }^{\dagger}$ Email address: arvind@physics.iisc.ernet.in

*Email address: scsp@uohyd.ernet.in

${ }^{\S}$ Email address: simon@imsc.ernet.in
}

structed out of two sets of orthonormal basis vectors. We investigate, in detail, their properties and identify and construct a full set of gauge-invariant building blocks for the $n$-level system. We also develop a canonical representation of $\mathrm{U}(n)$ matrices and bring out its relation to the Bargmann invariants. This representation has recently been shown to be extremely useful in parametrizing the Cabibbo-KobayashiMaskawa matrix that arises in the context of $C P$ violation in particle physics [10].

As noted above, the geometric phase becomes undefined for those open curves in the Hilbert space that have orthogonal vectors at their ends. A recent work by Manini and Pistolesi [11] addresses itself precisely to such exceptional cases. Employing the original Berry setting of adiabatic evolution for an $n$-level quantum system, they introduce the concept of off-diagonal geometric phases that can be meaningfully defined in such cases, and showed that these offdiagonal geometric phases play an essential role in the interpretation of the findings of a recent experiment [12].

We study the off-diagonal phases of Manini and Pistolesi within the framework of the quantum kinematic approach and show that, in actual fact, this approach, if suitably augmented, is robust enough to accommodate the off-diagonal phases as well. A brief outline of this work is as follows. In Sec. II, we quickly recapitulate the basic ingredients of the quantum kinematic approach to geometric phases in a general setting. In Sec. III, we specialize the discussion to $n$-level systems and construct the gauge-invariant objects for this case. These turn out to be $n$ geometric phases and a collection of four-vertex Bargmann invariants. Next we consider the problem of counting, leading to identification of a set of independent Bargmann invariants. This necessitates a detailed analysis of the structure of the $\mathrm{U}(n)$ matrix group. 
In Sec. IV, we develop a canonical representation for $\mathrm{U}(n)$ matrices that is then used in Sec. V for isolating the independent four-vertex Bargmann invariants. In Sec. IV, we apply the machinery developed in the previous sections to the offdiagonal geometric phases and show how they can be expressed in terms of the ordinary geometric phases and the four-vertex Bargmann invariants, thus liberating these phases from the assumptions of adiabaticity. Section VII contains our conclusions.

\section{GEOMETRIC PHASES AND BARGMANN INVARIANTS}

We review very briefly the background and ingredients that go into the definition of the quantum geometric phase from the kinematic viewpoint and then the Bargmann invariants and their properties in the generic case. Let $\mathcal{H}$ be the Hilbert space of states of some quantum system and let $\mathcal{B}$ be the set of unit vectors in $\mathcal{H}$,

$$
\mathcal{B}=\left\{\psi \in \mathcal{H} \mid\|\psi\|^{2}=(\psi, \psi)=1\right\} \subset \mathcal{H} .
$$

The corresponding ray space (consisting of equivalence classes of unit vectors that differ from one another by phases) and projection map are written as $\mathcal{R}$ and $\pi$, respectively,

$$
\pi: \quad \mathcal{B} \rightarrow \mathcal{R}=(\text { space of unit rays }) .
$$

To arrive at the concept of geometric phase, we begin with parametrized smooth (for our purposes continuous and once piecewise differentiable) curves $\mathcal{C}$ of unit vectors, which may be pictured as strings lying in $\mathcal{B}$,

$$
\mathcal{C}=\left\{\psi(s) \in \mathcal{B} \mid s_{1} \leqslant s \leqslant s_{2}\right\} \subset \mathcal{B} .
$$

A gauge transformation is a smooth change of phase in a parameter-dependent manner at each point of such a curve $\mathcal{C}$ to lead to another $\mathcal{C}^{\prime}$,

$$
\mathcal{C}^{\prime}=\left\{\psi^{\prime}(s)=e^{i \alpha(s)} \psi(s) \mid \psi(s) \in \mathcal{C}, s_{1} \leqslant s \leqslant s_{2}\right\} \subset \mathcal{B} .
$$

Then $\mathcal{C}^{\prime}$ and $\mathcal{C}$ share a common parametrized image curve $C$ in ray space,

$$
\pi\left[\mathcal{C}^{\prime}\right]=\pi[\mathcal{C}]=C \subset \mathcal{R} .
$$

In general, we permit $\mathcal{C}$ and even $C$ to be open curves.

The total, dynamical, and geometric phases are then defined as follows as functionals of appropriate arguments:

$$
\begin{gathered}
\varphi_{\text {tot }}[\mathcal{C}]=\arg \left[\psi\left(s_{1}\right), \psi\left(s_{2}\right)\right], \\
\varphi_{\text {dyn }}[\mathcal{C}]=\operatorname{Im} \int_{s_{1}}^{s_{2}} d s\left(\psi(s), \frac{d \psi(s)}{d s}\right), \\
\varphi_{g}[C]=\varphi_{\text {tot }}[\mathcal{C}]-\varphi_{\text {dyn }}[\mathcal{C}] .
\end{gathered}
$$

While the first two phases are functionals of $\mathcal{C}$ and do change under a gauge transformation, the geometric phase $\varphi_{g}[C]$ is gauge invariant, which explains why it is written as a func- tional of the ray-space curve $C$. All three phases are, however, individually reparametrization invariant.

Now we turn to the Bargmann invariants and their connection to geometric phases. Given any sequence of $n$ vectors $\chi_{1}, \chi_{2}, \ldots, \chi_{n}$ in $\mathcal{B}$, the corresponding $n$-vertex Bargmann invariant is

$$
\begin{aligned}
\boldsymbol{\Delta}_{n}\left(\chi_{1}, \chi_{2}, \ldots, \chi_{n}\right) \equiv & \left(\chi_{1}, \chi_{2}\right)\left(\chi_{2}, \chi_{3}\right) \cdots\left(\chi_{n-1}, \chi_{n}\right) \\
& \times\left(\chi_{n}, \chi_{1}\right) .
\end{aligned}
$$

Here we assume in the generic case that no two successive vectors in the sequence are mutually orthogonal. It is clear that this expression is invariant under cyclic permutations of the $\chi$ 's, and also under independent phase changes of the individual vectors. Therefore, it is actually a quantity defined at the ray-space level. It turns out that the phase of $\Delta_{n}\left(\chi_{1}, \chi_{2}, \ldots, \chi_{n}\right)$ is the geometric phase for suitably constructed closed ray-space curves obtained by joining each $\chi_{j}$ to the next $\chi_{j+1}$ (and finally $\chi_{n}$ to $\chi_{1}$ ) by any so-called "nullphase curve" [6]. A null-phase curve is a continuous ray-space curve such that for any finite connected portion of it the geometric phase vanishes. That is, "being in phase" in the Pancharatnam sense [13] becomes an equivalence relation on such curves. Examples of null-phase curves are rayspace geodesics (with respect to the well-known Fubinistudy metric $[14,15])$, but the former are a much larger set than the latter [6]. It should be emphasized that whereas the Bargmann invariant (7) is defined once its "vertices," namely, the projections $\pi\left(\chi_{1}\right), \pi\left(\chi_{2}\right), \ldots, \pi\left(\chi_{n}\right)$ in $\mathcal{R}$, are given, to interpret its phase as a geometric phase requires that we join each $\chi_{j}$ to the next $\chi_{j+1}$ in some definite manner, namely, by some null-phase curve, resulting in an " $n$-sided" closed figure in $\mathcal{R}$.

It can now be seen that as far as phases are concerned, an $n$-vertex Bargmann invariant for $n \geqslant 4$ can be reduced to a product of $\boldsymbol{\Delta}_{3}$ factors in the generic case [2], so we can regard the three-vertex Bargmann invariants as the primitive ones. For example, we have

$$
\begin{aligned}
& \boldsymbol{\Delta}_{4}\left(\chi_{1}, \chi_{2}, \chi_{3}, \chi_{4}\right) \\
& \quad=\boldsymbol{\Delta}_{3}\left(\chi_{1}, \chi_{2}, \chi_{3}\right) \boldsymbol{\Delta}_{3}\left(\chi_{1}, \chi_{3}, \chi_{4}\right) /\left.\left(\chi_{1}, \chi_{3}\right)\right|^{2},
\end{aligned}
$$

and more generally

$$
\begin{aligned}
& \boldsymbol{\Delta}_{n}\left(\chi_{1}, \chi_{2}, \ldots, \chi_{n}\right) \\
& \quad=\boldsymbol{\Delta}_{3}\left(\chi_{1}, \chi_{2}, \chi_{3}\right) \boldsymbol{\Delta}_{n-1}\left(\chi_{1}, \chi_{3}, \psi_{4}, \ldots, \chi_{n}\right) /\left|\left(\chi_{1}, \chi_{3}\right)\right|^{2} .
\end{aligned}
$$

Thus, the geometric phases of ray-space "triangles," each of whose sides is a null-phase curve, are primitive or irreducible phases, and all others can be built up from them additively. The purpose in mentioning this is that in the particular situation we shall be dealing with later the primitive Bargmann invariants will turn out to be $\boldsymbol{\Delta}_{4}$ 's rather than $\Delta_{3}$ 's, so that situation will not be generic in the present sense. 


\section{GAUGE-INVARIANT PHASES FOR $\boldsymbol{n}$-LEVEL SYSTEMS}

We now turn to a study of phases associated with $n$-level quantum systems. Thus we have an $n$-dimensional complex Hilbert space $\mathcal{H}_{n}$ describing the pure states of the system. The unit sphere in $\mathcal{H}_{n}$ and the corresponding space of unit rays will be denoted by $\mathcal{B}_{n}$ and $\mathcal{R}_{n}$, respectively.

If we imagine that a time-dependent Hamiltonian $(n \times n$ Hermitian matrix) is given, then at each time its complete orthonormal set of eigenvectors defines an orthonormal basis for $\mathcal{H}_{n}$. Assuming there are no degeneracies or level crossings, the eigenvalues can be arranged in, say, increasing order and at each time this basis for $\mathcal{H}_{n}$ is defined up to the freedom of phase changes in each basis vector. As time progresses this basis experiences a continuous unitary rotation.

In keeping with the approach of the previous section, however, we will adopt a kinematic approach here as well and not assume any particular Hamiltonian to be given. Thus, we imagine that for each value of a parameter $s$ in the range $s_{1} \leqslant s \leqslant s_{2}$, we have an orthonormal basis $\psi_{j}(s), j$ $=1,2, \ldots, n$ for $\mathcal{H}_{n}$; and as $s$ evolves this basis experiences a continuous unitary evolution. Thus we have

$$
\begin{gathered}
\left(\psi_{j}(s), \psi_{k}(s)\right)=\delta_{j k}, \quad j, k=1,2, \ldots, n, \\
\sum_{j=1}^{n} \psi_{j}(s) \psi_{j}(s)^{\dagger}=\mathcal{I}, \quad s_{1} \leqslant s \leqslant s_{2} .
\end{gathered}
$$

For ease in writing, we shall denote these vectors at the end points $s_{1}$ and $s_{2}$ as follows:

$$
\psi_{j}\left(s_{1}\right)=\psi_{j}, \quad \psi_{j}\left(s_{2}\right)=\phi_{j} .
$$

Our aim is to obtain gauge-invariant expressions and phases in this context. We expect to be able to construct both geometric phases $\varphi_{g}[C]$ for various $C$ and Bargmann invariants.

For each value of the index $j$, as $s$ varies from $s_{1}$ to $s_{2}$, the vector $\psi_{j}(s)$ traces out a particular continuous parametrized curve $\mathcal{C}_{j}$ in $\mathcal{B}_{n}$,

$$
\mathcal{C}_{j}=\left\{\psi_{j}(s) \in \mathcal{B}_{n} \mid s_{1} \leqslant s \leqslant s_{2}\right\} \subset \mathcal{B}_{n}, \quad j=1,2, \ldots, n .
$$

This curve runs from $\psi_{j}$ to $\phi_{j}$. Its image is $\pi\left[\mathcal{C}_{j}\right]$ $=C_{j} \subset \mathcal{R}_{n}$ and we have $n$ distinct geometric phases,

$$
\begin{gathered}
\varphi_{g}\left[C_{j}\right]=\varphi_{\text {tot }}\left[\mathcal{C}_{j}\right]-\varphi_{\text {dyn }}\left[\mathcal{C}_{j}\right] \\
\varphi_{\text {tot }}\left[\mathcal{C}_{j}\right]=\arg \left(\psi_{j}, \phi_{j}\right), \\
\varphi_{\text {dyn }}\left[\mathcal{C}_{j}\right]=\operatorname{Im} \int_{s_{1}}^{s_{2}} d s\left(\psi_{j}(s), \frac{d \psi_{j}(s)}{d s}\right), \quad j=1,2, \ldots, n .
\end{gathered}
$$

Each of these geometric phases is unchanged under arbitrary alterations in the phase of each $\psi_{j}(s)$ at each parameter value $s$.

We next turn to the construction of Bargmann invariants, the vertices of which are taken from the $n$ initial orthonormal vectors $\psi_{1}, \ldots, \psi_{n}$ and the $n$ final ones $\phi_{1}, \ldots, \phi_{n}$. Here we encounter an interesting difference compared to the generic situation discussed in the previous section. Since any two distinct $\psi_{j}$ 's (and similarly any two distinct $\phi_{j}$ 's) are orthogonal, in any Bargmann invariant $\boldsymbol{\Delta}_{n}(\cdots)$ an argument $\psi_{j}$ must be followed by an argument $\phi_{k}$, which must be followed by some $\psi_{\ell}$, and so on. Similarly, if the first argument is some $\psi$, the last one must be some $\phi$. Thus in the present context only even-order Bargmann invariants $\boldsymbol{\Delta}_{2 \ell}$ survive, a general one being

$$
\begin{aligned}
\Delta_{2} \lambda & \left(\psi_{j_{1}}, \phi_{k_{1}}, \psi_{j_{2}}, \phi_{k_{2}}, \ldots, \psi_{j \ell}, \phi_{k_{\ell}}\right) \\
= & \left(\psi_{j_{1}}, \phi_{k_{1}}\right)\left(\phi_{k_{1}}, \psi_{j_{2}}\right) \\
& \times\left(\psi_{j_{2}}, \phi_{k_{2}}\right) \cdots\left(\psi_{j}, \phi_{k}\right)\left(\phi_{k_{\ell}}, \psi_{j_{1}}\right) .
\end{aligned}
$$

We may now regard the generic case as obtaining when every inner product $\left(\psi_{j}, \phi_{k}\right)$ is nonzero. In this situation we find that the primitive Bargmann invariants are $\boldsymbol{\Delta}_{4}$ 's rather than $\boldsymbol{\Delta}_{3}$ 's, for instance,

$$
\begin{aligned}
\Delta_{6}\left(\psi_{j_{1}}, \phi_{k_{1}}, \psi_{j_{2}}, \phi_{k_{2}}, \psi_{j_{3}}, \phi_{k_{3}}\right) \\
=\Delta_{4}\left(\psi_{j_{1}}, \phi_{k_{1}}, \psi_{j_{2}}, \phi_{k_{2}}\right) \\
\quad \times \Delta_{4}\left(\psi_{j_{1}}, \phi_{k_{2}}, \psi_{j_{3}}, \phi_{k_{3}}\right) /\left|\left(\psi_{j_{1}}, \phi_{k_{2}}\right)\right|^{2},
\end{aligned}
$$

and similarly for higher-order $\boldsymbol{\Delta}_{2}$ 's.

From this discussion, it emerges that in the present context the basic gauge-invariant expressions are the $n$ geometric phases $\varphi_{g}\left[C_{j}\right]$ and the various four-vertex Bargmann invariants $\boldsymbol{\Delta}_{4}\left(\psi_{j_{1}}, \phi_{k_{1}}, \psi_{j_{2}}, \phi_{k_{2}}\right)$. (Here we are using quantities referring to the entire parameter range $s_{1} \leqslant s \leqslant s_{2}$ and to its end points and not to any subranges.) An important problem that now remains is to select out of all possible $\boldsymbol{\Delta}_{4}$ 's a maximal set of independent ones as far as phases are concerned. For this, as a first step, we turn to an interesting analysis of the structure of the unitary matrix groups $\mathrm{U}(n)$.

\section{A CANONICAL REPRESENTATION FOR U $(\boldsymbol{n})$ MATRICES, COUNTING OF INVARIANT PHASES}

Referring to Eqs. (10) and (11), we have a parameterdependent $n \times n$ unitary matrix describing the transition from the initial orthonormal basis $\left\{\psi_{j}\right\}$ for $\mathcal{H}_{n}$ at $s=s_{1}$ to the moving basis $\left\{\psi_{j}(s)\right\}$ at a general $s$,

$$
\begin{gathered}
A(s)=a_{j k}(s) \in U(n), \\
a_{j k}(s)=\left(\psi_{j}, \psi_{k}(s)\right), \quad s_{1} \leqslant s \leqslant s_{2}, \\
a_{j k}\left(s_{1}\right)=\delta_{j k} .
\end{gathered}
$$

At $s=s_{2}$ we write $A\left(s_{2}\right)=A$,

$$
\begin{gathered}
A=\left(a_{j k}\right), \\
a_{j k}=\left(\psi_{j}, \phi_{k}\right) .
\end{gathered}
$$


The four-vertex Bargmann invariants of the type appearing in Eq. (15) are expressions involving products of matrix elements of $A$ and their complex conjugates,

$$
\begin{aligned}
\boldsymbol{\Delta}_{4}\left(\psi_{j}, \phi_{k}, \psi_{\ell}, \phi_{m}\right) & =\left(\psi_{j}, \phi_{k}\right)\left(\phi_{k}, \psi_{\ell}\right)\left(\psi_{\ell}, \phi_{m}\right)\left(\phi_{m}, \psi_{j}\right) \\
& =a_{j k} a_{\ell k}^{*} a_{\ell m} a_{j m}^{*} .
\end{aligned}
$$

Our problem is to determine how many algebraically independent $\boldsymbol{\Delta}_{4}$ 's are there in the generic case insofar as their phases are concerned and to find a convenient enumeration of them. This turns out to be a somewhat intricate problem. After some preparation in this section, the solution will be developed in the next one.

In working with $n \times n$ unitary matrices it is convenient to keep in mind the standard basis in $\mathcal{H}_{n}$. Then $\mathrm{U}(n)$ is the group of unitary transformations acting on all $n$ dimensions. For $m=1,2, \ldots, n-1$, we will denote by $\mathrm{U}(m)$ the unitary group acting on the first $m$ dimensions in $\mathcal{H}_{n}$, leaving dimensions $m+1, m+2, \ldots, n$ unaffected. Then we have the inclusion relations (the canonical subgroup chain)

$$
\mathrm{U}(1) \subset \mathrm{U}(2) \subset \mathrm{U}(3) \cdots \subset \mathrm{U}(n-1) \subset \mathrm{U}(n) .
$$

General matrices of $\mathrm{U}(n), \mathrm{U}(n-1), \ldots$ will be written as $A_{n}, A_{n-1}, \ldots$. In a matrix $A_{m} \in \mathrm{U}(m)$, the last $(n-m)$ rows and columns are trivial, with ones along the diagonal and zeros elsewhere. (When no confusion is likely to arise, $A_{m}$ will also denote an unbordered $m \times m$ unitary matrix.)

We will now show by a recursive argument that (almost all) elements $A_{n} \in \mathrm{U}(n)$ can be expressed uniquely as $n$-fold products,

$A_{n}=A_{n}(\underline{\zeta}) A_{n-1}\left(\underline{\eta)} A_{n-2}(\underline{\xi}) \cdots A_{3}(\beta) A_{2}(\alpha) A_{1}(\chi)\right.$,

where $A_{n}(\zeta)$ is a special $\mathrm{U}(n)$ element determined by an $n$-component complex unit vector $\underline{\zeta} \in \mathcal{B}_{n} ; A_{n-1}(\underline{\eta})$ is a special $\mathrm{U}(n-1)$ element determined by an $(n-1)$-component complex unit vector $\eta \in \mathcal{B}_{n-1}$; and so on down to $A_{2}(\underline{\alpha})$ that is a special $\mathrm{U}(2)$ element determined by a two-component complex unit vector $\underline{\alpha} \in \mathcal{B}_{2}$ and $A_{1}(\chi)$ is a phase factor belonging to $\mathrm{U}(1)$. We are led to expect such a representation for $A_{n}$ by the following argument. Any vector $\zeta \in \mathcal{B}_{n}$ can be carried by a suitable $\mathrm{U}(n)$ element into the $n$th vector of the standard basis, $(0,0, \ldots, 0,1)^{T}$ and the stability group of this vector is the subgroup $\mathrm{U}(n-1) \subset \mathrm{U}(n)$ acting on the first $(n-1)$ dimensions in $\mathcal{H}_{n}$. Thus $\mathrm{U}(n)$ acts transitively on $\mathcal{B}_{n}$ and this is just the coset space $\mathrm{U}(n) / \mathrm{U}(n-1)$. Each coset is thus uniquely labeled by some $\underline{\zeta} \in \mathcal{B}_{n}$. We, therefore, expect that a general $A_{n} \in \mathrm{U}(n)$ is expressible as the product $A_{n}(\underline{\zeta}) A_{n-1}$ where $\underline{\zeta}$ is the last column in $A_{n}$ and $A_{n}(\underline{\zeta})$ is a suitably chosen coset representative. Repeating this argument $(n-1)$ times we are led to expect the representation (20). The counting of parameters is also just right. Remembering that $\underline{\alpha}, \underline{\beta}, \ldots, \underline{\xi}, \underline{\eta}, \underline{\zeta}$ are complex unit vectors of dimensions $2,3, \ldots, n-2, n-1, n$ and adding the $\mathrm{U}(1)$ phase $\chi$, the number of real independent parameters adds up to $n^{2}$, the dimension of $\mathrm{U}(n)$.
We now present the argument leading to Eq. (20), yielding in the process the determination of $A_{n}(\zeta)$. Let a generic matrix $A_{n}=\left(a_{j k}\right) \in \mathrm{U}(n)$ be given and let its last ( $n$ th) column be $\underline{\zeta}$,

$$
a_{j n}=\zeta_{j}, \quad j=1,2, \ldots, n .
$$

Multiplying $A_{n}$ by an $A_{n-1}$ on the right leaves this column unchanged. We choose $A_{n-1}$ so as to bring the $n$th row of $A_{n}$ to a particularly simple form (for ease in writing we keep using $A_{n}$ and $a_{j k}$ for the $\mathrm{U}(n)$ element obtained at each successive stage of the argument),

$$
\begin{gathered}
a_{n k}=0, k=1,2, \ldots, n-2, \\
a_{n, n-1}=\text { real positive }=\left(1-\left|\zeta_{n}\right|^{2}\right)^{1 / 2} .
\end{gathered}
$$

The $A_{n-1}$ used here is arbitrary up to an $A_{n-2}$ factor on its right. Having simplified the $n$th row of $A_{n}$ in this way, we can determine all the other elements in the $(n-1)$ th column by imposing orthogonality of rows $1,2, \ldots, n-1$ to row $n$,

$$
a_{n, n-1} a_{j, n-1}=-\zeta_{n}^{*} \zeta_{j}, \quad j=1,2, \ldots, n-1 .
$$

At this point the last two columns and the last row of $A_{n}$ are known in terms of $\zeta$.

We next use the freedom in choice of $A_{n-1}$ mentioned above and multiply $A_{n}$ on the right by a suitable $A_{n-2}$ (unique up to an $A_{n-3}$ on its right) to bring the (n-1)th row of $A_{n}$ to a particularly simple form,

$$
\begin{gathered}
a_{n-1, k}=0, \quad k=1,2, \ldots, n-3, \\
a_{n-1, n-2}=\text { real positive. }
\end{gathered}
$$

Normalizing this row gives $a_{n-1, n-2}$,

$$
a_{n, n-1} a_{n-1, n-2}=\left(1-\left|\zeta_{n}\right|^{2}-\left|\zeta_{n-1}\right|^{2}\right)^{1 / 2} .
$$

Next we determine all the remaining elements in the ( $n$ -2)th column of $A_{n}$ by imposing orthogonality of rows $1,2, \ldots, n-2$ to row $(n-1)$,

$$
a_{n, n-1}^{2} a_{n-1, n-2} a_{j, n-2}=-\zeta_{n-1}^{*} \zeta_{j}, \quad j=1,2, \ldots, n-2 .
$$

At this point the last three columns and last two rows of $A_{n}$ are known in terms of $\underline{\zeta}$.

This argument can be repeated all the way until we obtain a matrix $A_{n}(\underline{\zeta}) \in \mathrm{U}(n)$, all of whose elements are determined by the $n$th column $\zeta$, namely, it serves as a coset representative in the coset space $\mathrm{U}(n) / \mathrm{U}(n-1)$. (In particular, the last $\mathrm{U}(1)$ element $A_{1}(\chi)$ is used to make $a_{21}$ real positive). After some algebra, we obtain the result that the matrix $A_{n}(\underline{\zeta})=a_{j k}(\underline{\zeta})$ is uniquely determined by the conditions 


$$
\begin{gathered}
a_{j k}(\underline{\zeta})=0, \quad j \geqslant k+2, \\
a_{j, j-1}(\underline{\zeta})=\text { real positive }, \quad j=2,3, \ldots, n, \\
a_{j n}(\underline{\zeta})=\zeta_{n}, \quad j=1,2, \ldots, n .
\end{gathered}
$$

Thus $A_{n}(\underline{\zeta})$ has vanishing matrix elements in the lower left hand triangular portion up to two steps below the main diagonal, nonzero matrix elements appear only one step below the main diagonal, and beyond. The explicit expressions for the nonzero matrix elements are

$$
\begin{gathered}
a_{j, j-1}(\underline{\zeta})=\rho_{j-1} / \rho_{j}, \quad j=2,3, \ldots, n, \\
a_{j, k}(\underline{\zeta})=-\zeta_{k+1}^{*} \zeta_{j} / \rho_{k} \rho_{k+1}, \quad j \leqslant k \leqslant n-1, \\
a_{j n}(\underline{\zeta})=\zeta_{n}, \quad j=1,2, \ldots, n, \\
\rho_{j}=\left(\left|\zeta_{1}\right|^{2}+\left|\zeta_{2}\right|^{2}+\ldots+\left|\zeta_{j}\right|^{2}\right)^{1 / 2} \\
=\left(1-\left|\zeta_{j+1}\right|^{2}-\left|\zeta_{j+2}\right|^{2}-\ldots-\left|\zeta_{n}\right|^{2}\right)^{1 / 2} .
\end{gathered}
$$

Since the quantities $\rho_{j}$ obey

$$
\rho_{1}=\left|\zeta_{1}\right| \leqslant \rho_{2} \leqslant \rho_{3} \leqslant \cdots \leqslant \rho_{n-1} \leqslant \rho_{n}=1,
$$

it is evident that this determination of $A_{n}(\zeta)$ goes through with no problems as long as $\zeta_{1}$ is nonzero, i.e., $\rho_{1}>0$.

It may be helpful to give the expressions for $A_{2}(\underline{\alpha})$ $\in \mathrm{U}(2), A_{3}(\underline{\beta}) \in \mathrm{U}(3)$ determined in this way, so as to see the general pattern,

$$
\begin{gathered}
A_{2}(\underline{\alpha})=\left(\begin{array}{cc}
\frac{-\alpha_{2}^{*} \alpha_{1}}{\left|\alpha_{1}\right|} & \alpha_{1} \\
\left|\alpha_{1}\right| & \alpha_{2}
\end{array}\right), \quad\left|\alpha_{1}\right|^{2}+\left|\alpha_{2}\right|^{2}=1, \\
A_{3}(\underline{\beta})=\left(\begin{array}{ccc}
\frac{-\beta_{2}^{*} \beta_{1}}{\rho_{1} \rho_{2}} & \frac{-\beta_{3}^{*} \beta_{1}}{\rho_{2}} & \beta_{1} \\
\rho_{1} / \rho_{2} & \frac{-\beta_{3}^{*} \beta_{2}}{\rho_{2}} & \beta_{2} \\
0 & \rho_{2} & \beta_{3}
\end{array}\right) \\
\left|\beta_{1}\right|^{2}+\left|\beta_{2}\right|^{2}+\left|\beta_{3}\right|^{2}=1, \\
\rho_{1}=\left|\beta_{1}\right|, \quad \rho_{2}=\left(1-\left|\beta_{3}\right|^{2}\right)^{1 / 2} .
\end{gathered}
$$

We notice in passing that these are not elements of SU(2) and $\mathrm{SU}(3)$, respectively.

Going back to the proof of Eq. (20), we see that it can be recursively established, and $\chi, \underline{\alpha}, \underline{\beta}, \ldots, \underline{\xi}, \underline{\eta}, \underline{\zeta}$ supply us with exactly $n^{2}$ real independent parameters for $A_{n}$. Of these, the $\frac{1}{2} n(n-1)$ independent quantities $\left|\alpha_{1}\right|,\left|\beta_{1}\right|,\left|\beta_{2}\right|, \ldots,\left|\zeta_{1}\right|,\left|\zeta_{2}\right|, \ldots,\left|\zeta_{n-1}\right|$ are of the modulus type and then there are $\frac{1}{2} n(n+1)$ independent phases. We can display a general element $A_{n} \in \mathrm{U}(n)$ [in particular, $A$ of Eq. (17)] in the self-evident forms

$$
\begin{gathered}
A_{n}=A_{n}(\underline{\zeta}, \underline{\eta}, \underline{\xi}, \ldots, \underline{\beta}, \underline{\alpha}, \chi)=A_{n}(\underline{\zeta}) A_{n-1}, \\
A_{n-1}=A_{n-1}(\underline{\eta}, \underline{\xi}, \ldots, \underline{\beta}, \underline{\alpha}, \chi) .
\end{gathered}
$$

We are now interested in the following operation: suppose we premultiply and post multiply $A_{n}$ by two independent diagonal elements of $\mathrm{U}(n)$ (a "gauge transformation" of $\left.A_{n}\right)$,

$$
\begin{gathered}
A_{n} \rightarrow A_{n}^{\prime}=D_{n}\left(\theta_{1}, \theta_{2}, \ldots, \theta_{n}\right) A_{n} D_{n}\left(\theta_{1}^{\prime}, \theta_{2}^{\prime}, \ldots, \theta_{n}^{\prime}\right), \\
D_{n}\left(\theta_{1}, \theta_{2}, \ldots, \theta_{n}\right)=\operatorname{diag}\left(e^{i \theta_{1}}, e^{i \theta_{2}}, \ldots, e^{i \theta_{n}}\right) .
\end{gathered}
$$

We would like to know: how many independent invariants can we construct out of $A_{n}$ under these transformations, how many of them are phases, and how can they be captured through four-vertex Bargmann invariants? In the case of the matrix $A$ of Eq. (17) the transformation (32) amounts to changing the phase of each $\psi_{j}$ and each $\phi_{k}$ independently,

$$
\begin{gathered}
\psi_{j}^{\prime}=e^{-i \theta_{j}} \psi_{j}, \\
\phi_{k}^{\prime}=e^{i \theta_{k}^{\prime}} \phi_{k}, \\
a_{j k}^{\prime}=e^{i\left(\theta_{j}+\theta_{k}^{\prime}\right)} a_{j k},
\end{gathered}
$$

and we seek an independent set of invariant expressions of the form (18).

First we count the expected numbers of invariants of each kind. The real dimension of $\mathrm{U}(n)$ is $n^{2}$. The number of independent $\theta$ 's and $\theta^{\prime}$ 's in [Eq. (32)] is $(2 n-1)$ because an overall constant phase can be attributed to either the left or the right diagonal factor. Therefore, the number of real invariants is $(n-1)^{2}$. In the description (31) of a general $A_{n}$ $\in \mathrm{U}(n)$, it is clear that under [Eq. (32)] every component of each of $\underline{\alpha}, \underline{\beta}, \ldots, \zeta$ just undergoes a phase change, so the quantities $\left|\alpha_{1}\right|,\left|\beta_{1}\right|,\left|\beta_{2}\right|, \ldots,\left|\zeta_{1}\right|,\left|\zeta_{2}\right|, \ldots,\left|\zeta_{n-1}\right|$ are $\frac{1}{2} n(n-1)$ real independent modulus-type invariants. Thus there must be a balance of $\frac{1}{2}(n-1)(n-2)$ real independent phase-type invariants. This is in agreement with known results [16].

We shall describe in the next section a recursive procedure by which we can pick out $\frac{1}{2}(n-1)(n-2)$ algebraically independent four-vector Bargmann invariants whose phases are the expected phase invariants associated with a general $\mathrm{U}(n)$ matrix.

\section{DETERMINATION OF INDEPENDENT BARGMANN INVARIANTS}

We first describe how, in a recursive manner, we can isolate the expected $\frac{1}{2}(n-1)(n-2)$ independent gaugeinvariant phases for a generic $A_{n} \in \mathrm{U}(n)$ using the parametrization (20), and then turn to the choice of an equal number of independent primitive Bargmann invariants $\boldsymbol{\Delta}_{4}$.

We begin with Eqs. (20) and (31), 


$$
\begin{gathered}
A_{n}=A_{n}(\underline{\zeta}) A_{n-1}, \\
A_{n-1}=A_{n-1}(\underline{\eta}) A_{n-2}(\underline{\xi}) \cdots A_{2}(\underline{\alpha}) A_{1}(\chi) \\
=A_{n-1}(\underline{\eta}, \underline{\xi}, \ldots, \underline{\alpha}, \chi),
\end{gathered}
$$

apply diagonal matrices on the left and on the right as in Eq. (32), and trace the changes that occur in $\zeta$ and in $A_{n-1}$,

$$
\begin{aligned}
A_{n}^{\prime} & =D_{n}\left(\theta_{1}, \theta_{2}, \ldots, \theta_{n}\right) A_{n} D_{n}\left(\theta_{1}^{\prime}, \theta_{2}^{\prime}, \ldots, \theta_{n}^{\prime}\right) \\
& =A_{n}\left(\zeta^{\prime}\right) A_{n-1}^{\prime} .
\end{aligned}
$$

Our aim is to compute $\zeta^{\prime}$ and $A_{n-1}^{\prime}$. Since the $D_{n}$ factors are quite elementary, this can be carried through as follows:

$$
\begin{aligned}
A_{n}^{\prime}= & D_{n}\left(\theta_{1}, \theta_{2}, \ldots, \theta_{n}\right) A_{n}(\underline{\zeta}) A_{n-1} D_{n}\left(\theta_{1}^{\prime}, \theta_{2}^{\prime}, \ldots, \theta_{n}^{\prime}\right) \\
= & D_{n}\left(\theta_{1}, \theta_{2}, \ldots, \theta_{n}\right) A_{n}(\underline{\zeta}) A_{n-1} D_{n}\left(0,0, \ldots, 0, \theta_{n}^{\prime}\right) \\
& \times D_{n}\left(\theta_{1}^{\prime}, \theta_{2}^{\prime}, \ldots, \theta_{n-1}^{\prime}, 0\right) \\
= & D_{n}\left(\theta_{1}, \theta_{2}, \ldots, \theta_{n}\right) A_{n}(\underline{\zeta}) D_{n}\left(0, \ldots, 0, \theta_{n}^{\prime}\right) A_{n-1} \\
& \times D_{n-1}\left(\theta_{1}^{\prime}, \theta_{2}^{\prime}, \ldots, \theta_{n-1}^{\prime}\right) .
\end{aligned}
$$

The product of the first three factors simplifies as

$$
\begin{aligned}
D_{n}\left(\theta_{1}, \theta_{2}, \ldots, \theta_{n}\right) A_{n}(\underline{\zeta}) D_{n}\left(0, \ldots, 0, \theta_{n}^{\prime}\right) \\
=D_{n}\left(\theta_{1}, \theta_{2}, \ldots, \theta_{n}\right)\left(a_{j k}(\underline{\zeta})\right) D_{n}\left(0,0, \ldots, \theta_{n}^{\prime}\right) \\
=b_{j k}(\underline{\zeta}), \\
b_{j n}(\underline{\zeta})=\zeta_{j}^{\prime}=e^{i\left(\theta_{j}+\theta_{n}^{\prime}\right)} \zeta_{j}, \\
b_{j k}(\underline{\zeta})=e^{i \theta_{j}} a_{j k}(\underline{\zeta}), \quad k=1,2, \ldots, n-1 .
\end{aligned}
$$

Here the matrix elements $a_{j k}(\underline{\zeta})$ are given in Eq. (28) and for simplicity the $\theta$ and $\theta^{\prime}$ dependences of $b_{j k}$ are left implicit. In particular, as in Eq. (27),

$$
b_{j k}(\underline{\zeta})=0, \quad k=1,2, \ldots, j-2 ; \quad j=3,4, \ldots, n,
$$

while

$b_{j, j-1}(\underline{\zeta})=e^{i \theta_{j}} a_{j, j-1}(\underline{\zeta})=e^{i \theta_{j}} \rho_{j-1} / \rho_{j}, \quad j=2,3, \ldots, n$.

Thus the matrix $b_{j k}(\underline{\zeta})$ would have been $a_{j k}\left(\underline{\zeta}^{\prime}\right)$ except for the fact that the elements $b_{j, j-1}(\zeta)$ just below the main diagonal are not real positive but carry phases. But this can be easily taken care of by extracting a suitably chosen diagonal matrix on the right,

$$
b_{j k}(\underline{\zeta})=\left(a_{j k}\left(\underline{\zeta}^{\prime}\right)\right) D_{n}\left(\theta_{2}, \theta_{3}, \ldots, \theta_{n}, 0\right) .
$$

The point is that, according to the statement accompanying Eq. (27), after removal of this diagonal factor what remains is necessarily $A_{n}\left(\underline{\zeta}^{\prime}\right)=a_{j k}\left(\underline{\zeta}^{\prime}\right)$. Combining the above steps we get

$$
\begin{aligned}
A_{n}^{\prime}= & A_{n}\left(\underline{\zeta}^{\prime}\right) A_{n-1}^{\prime} \\
= & \left(b_{j k}(\underline{\zeta})\right) A_{n-1} D_{n-1}\left(\theta_{1}^{\prime}, \theta_{2}^{\prime}, \ldots, \theta_{n-1}^{\prime}\right) \\
= & A_{n}\left(\underline{\zeta}^{\prime}\right) D_{n}\left(\theta_{2}, \theta_{3}, \ldots, \theta_{n}, 0\right) \\
& \times A_{n-1} D_{n-1}\left(\theta_{1}^{\prime}, \theta_{2}^{\prime}, \ldots, \theta_{n-1}^{\prime}\right),
\end{aligned}
$$

so the changes induced in $\zeta$ and in $A_{n-1}$ by the gauge transformation (35) are

$$
\begin{gathered}
\zeta_{j}^{\prime}=e^{i\left(\theta_{j}+\theta_{n}^{\prime}\right)} \zeta_{j}, \quad j=1,2, \ldots, n, \\
A_{n-1}^{\prime}=D_{n-1}\left(\theta_{2}, \theta_{3}, \ldots, \theta_{n}\right) A_{n-1} D_{n-1}\left(\theta_{1}^{\prime}, \theta_{2}^{\prime}, \ldots, \theta_{n-1}^{\prime}\right) .
\end{gathered}
$$

We see from the structure of this result that we can tackle our problem recursively. The gauge transformation (35) at the $\mathrm{U}(n)$ level translates into the change $\underline{\zeta} \rightarrow \underline{\zeta}^{\prime}$ given by Eq. (42a) and a gauge transformation $A_{n-1} \rightarrow \bar{A}_{n-1}^{\prime}$ at the $\mathrm{U}(n$ -1 ) level given by Eq. (42b). Therefore, all gauge-invariant expressions that exist at the $A_{n-1}$ or $\mathrm{U}(n-1)$ level survive when we move from $\mathrm{U}(n-1)$ to $\mathrm{U}(n)$ and in addition as the vector $\underline{\zeta} \in \mathcal{B}_{n}$ becomes available, new invariant phases involving $\zeta$ can be constructed. The number of the latter can be immediately computed: it is the difference between $\frac{1}{2}(n$ $-1)(n-2)$ and $\frac{1}{2}(n-2)(n-3)$, namely, the difference between the numbers of gauge-invariant phases at the $\mathrm{U}(n)$ and the $\mathrm{U}(n-1)$ levels, and this is $(n-2)$. Therefore, the number of new independent phase invariants involving $A_{n}(\underline{\zeta})$, i.e., $\zeta$, in an essential way must be $(n-2)$. These can now be isolated or explicitly constructed as follows.

From Eq. (42) we notice that $\theta_{1}$ and $\theta_{n}^{\prime}$ appears only in the transformation law for $\underline{\zeta}$, not for $A_{n-1}$. Therefore, we first form the $(n-1)$ independent combinations $\zeta_{j}^{*} \zeta_{j+1}$ to eliminate $\theta_{n}^{\prime}$ completely:

$$
\zeta_{j}^{*} \zeta_{j+1} \rightarrow \exp \left[-i\left(\theta_{j}-\theta_{j+1}\right)\right] \zeta_{j}^{*} \zeta_{j+1}, \quad j=1,2, \ldots, n-1 .
$$

Here $\theta_{1}$ occurs only in the transformation law for $\zeta_{1}^{*} \zeta_{2}$, being absent as we have just mentioned in the law for $A_{n-1}$. Next, we notice that the phases $\theta_{1}^{\prime}, \theta_{2}^{\prime}, \ldots, \theta_{n-1}^{\prime}$, involved in $A_{n-1}^{\prime}$, are completely absent in the transformation law (43) of $\zeta_{j}^{*} \zeta_{j+1}$. Let us, therefore, look at the $(n-1)$ th column, say, of $A_{n-1}$, which, as is evident from Eq. (34), is just the $(n-1)$ component complex unit vector $\eta \in \mathcal{B}_{n-1}$ :

$$
A_{n-1}=\left(\begin{array}{cccc}
\cdots & \cdots & \eta_{1} & 0 \\
\cdots & \ldots & \eta_{2} & 0 \\
\cdots & \ldots & \eta_{n-1} & 0 \\
0 & \ldots & 0 & 1
\end{array}\right) \text {. }
$$


The "earlier" columns of $A_{n-1}$ are more complicated, as is clear from the structure of $A_{n-1}$ in Eq. (34). From Eq. (42b) we can read off the transformation law for the $\eta$ 's under the gauge transformation (35):

$$
\eta_{j}^{\prime}=e^{i\left(\theta_{j+1}+\theta_{n-1}^{\prime}\right)} \eta_{j}, \quad j=1,2, \ldots, n-1 .
$$

To eliminate $\theta_{n-1}^{\prime}$ we form the $(n-2)$ combinations $\eta_{j} \eta_{j+1}^{*}$ that transform thus,

$$
\eta_{j} \eta_{j+1}^{*} \rightarrow e^{-i\left(\theta_{j+2}-\theta_{j+1}\right)} \eta_{j} \eta_{j+1}^{*}, \quad j=1,2, \ldots, n-2 .
$$

Comparing Eqs. (43) and (46) we immediately obtain the expected $(n-2)$ independent (phase-type) invariants involving $\underline{\zeta} \in \mathcal{B}_{n}$ in an essential manner, namely, they can be taken to be the complex quantities

$$
\eta_{j} \eta_{j+1}^{*} \zeta_{j+1}^{*} \zeta_{j+2}, \quad j=1,2, \ldots, n-2 .
$$

By recursion the complete set of $\frac{1}{2}(n-1)(n-2)$ independent phase-type invariants that can be formed from a generic matrix $A_{n} \in \mathrm{U}(n)$ can be written down in terms of the canonical parametrization (20) for $A_{n}$, and the list reads

$$
\begin{gathered}
\alpha_{j} \alpha_{j+1}^{*} \beta_{j+1}^{*} \beta_{j+2}, \quad j=1, \\
\beta_{j} \beta_{j+1}^{*} \gamma_{j+1}^{*} \gamma_{j+2}, \quad j=1,2, \\
\vdots \\
\xi_{j} \xi_{j+1}^{*} \eta_{j+1}^{*} \eta_{j+2}, \quad j=1,2, \ldots, n-3, \\
\eta_{j} \eta_{j+1}^{*} \zeta_{j+1}^{*} \zeta_{j+2}, \quad j=1,2, \ldots, n-2 .
\end{gathered}
$$

While here we have an explicit solution to our problem, the difficulty is that these invariants are not directly expressed in terms of the matrix elements of $A_{n}=\left(a_{j k}\right)$ $\in \mathrm{U}(n)$. It is true that in our parametrization $\zeta$ is the last, $n$ th, column of $A_{n}$; but the previous, $(n-1) \mathrm{t}$ column involves both $\underline{\eta}$ and $\underline{\xi}$; the $(n-2)$ th column involves $\underline{\xi}, \underline{\eta}$, and $\underline{\zeta}$, and so on. The task that remains is to see how to translate the expressions (48), as far as their phases are concerned, into an algebraically equivalent set of $\frac{1}{2}(n-1)(n-2)$ expressions formed as simply as possible out of the matrix elements of $A_{n}$. We turn to this now, bringing in the fourvertex Bargmann invariants of $A_{n}$.

As indicated in Eq. (18), a general four-vertex Bargmann invariant requires the choice of some two rows, say $j$ and $\ell$ with $j<\ell$, and some two columns, say $k$ and $m$ with $k<m$, and use of the four matrix elements at their intersections,

$$
\boldsymbol{\Delta}_{j \ell k m} \equiv a_{j k} a_{\ell k}^{*} a_{\ell m} a_{j m}^{*}
$$

But as far as phases go, we can see in a step by step manner that a general $\boldsymbol{\Delta}_{j \ell \mathrm{km}}$ reduces to a product of factors of the simpler form,

$$
\boldsymbol{\Delta}_{j k} \equiv \boldsymbol{\Delta}_{j, j+1, k, k+1},
$$

involving some two adjacent rows and some two adjacent columns. This is to be understood modulo real positive definite factors coming from the squared moduli of some of the matrix elements of $A_{n}$. The two "recursion formulas" that help us achieve this simplification are

$$
\begin{aligned}
\boldsymbol{\Delta}_{j \ell k m} & =\boldsymbol{\Delta}_{j \ell k m-1} \boldsymbol{\Delta}_{j \ell m-1 m} /\left|a_{j, m-1} a_{\ell, m-1}\right|^{2}, \\
& =\boldsymbol{\Delta}_{j \ell-1 k m} \boldsymbol{\Delta}_{\ell-1 \ell k m} /\left|a_{\ell-1, k} a_{\ell-1, m}\right|^{2} .
\end{aligned}
$$

It, therefore, suffices to work with the $(n-1)^{2}$ expressions

$\boldsymbol{\Delta}_{j k}=a_{j k} a_{j+1, k}^{*} a_{j+1, k+1} a_{j, k+1}^{*}, \quad j, k=1,2, \ldots, n-1$,

and their phases. Our goal now is to (at least in principle and in the generic situation) express (the phases of) the $\frac{1}{2}(n$ $-1)(n-2)$ complex invariants (48) in terms of (the phases of) the $(n-1)^{2}$ complex invariants (52). (In this process any number of real positive factors may intervene). Here we already have an indication that the $(n-1)^{2}$ expressions $(52)$ (more exactly their phases) cannot all be independent, the number of independent ones being only $\frac{1}{2}(n-1)(n-2)$. It will turn out, as we indicate below, that these may be taken to be the $\boldsymbol{\Delta}_{j k}$ for $j<k \leqslant n-1$. Again the proof is recursive in nature.

Consider the $(n-1)$ invariants $(47)$ that get added to all previous ones when we make the transition $\mathrm{U}(n-1)$ $\rightarrow \mathrm{U}(n)$ and bring in the vector $\zeta \in \mathcal{B}_{n}$. Instead of being expressed in terms of $\underline{\zeta}$ and $\underline{\eta} \in \overline{\mathcal{B}}_{n-1}$, we now show that they can be equally well expressed in terms of $\zeta$ and the penultimate, i.e., $(n-1)$ th column of the complete $\overline{\mathrm{U}}(n)$ matrix $A_{n}$. Let us denote this column vector by $\underline{w} \in \mathcal{B}_{n}$; it is orthogonal to $\underline{\zeta}$. As noted earlier, it is easily determined in terms of $\underline{\zeta}$ and $\underline{\eta}$ or, more conveniently for our purpose, $\underline{\eta}$ is expressible in terms of $\underline{w}$ and $\underline{\zeta}$. Starting with

$$
A_{n}=\left(\begin{array}{cccc}
\cdots & \cdots & w_{1} & \zeta_{1} \\
\cdots & \cdots & w_{2} & \zeta_{2} \\
\cdots & \cdots & w_{n} & \zeta_{n}
\end{array}\right)=A_{n}(\underline{\zeta}) A_{n-1}(\underline{\eta}) A_{n-2},
$$

and transposing $A_{n}(\underline{\zeta})$ we get

$$
A_{n-1}(\underline{\eta}) A_{n-2}=A_{n}(\underline{\zeta})^{\dagger} A_{n} .
$$

Since the factor $A_{n-2}$, does not affect the last two columns on both sides, we can use the matrix elements (27) and (28) of $A_{n}(\underline{\zeta})$ to obtain

$$
\begin{aligned}
\eta_{j}= & \sum_{k=1,2, \ldots}^{j+1} a_{k j}(\underline{\zeta})^{*} w_{k}=\frac{\rho_{j}}{\rho_{j+1}} w_{j+1} \\
& -\frac{\zeta_{j+1}}{\rho_{j} \rho_{j+1}} \sum_{k=1}^{j} \zeta_{j}^{*} w_{j}, \quad j=1,2, \ldots, n-1 .
\end{aligned}
$$

The gauge transformation laws of $\underline{\zeta}$ and $\underline{\eta}$ are given in Eqs. (42a) and (45), while that of $\underline{w}$ is seen from Eq. (35) to be 


$$
w_{j} \rightarrow e^{i\left(\theta_{j}+\theta_{n-1}^{\prime}\right)} w_{j}, \quad j=1,2, \ldots, n .
$$

Naturally the relations (55) are consistent with these transformation laws. The combinations of $\eta$ and $\zeta$ needed in (47) are $\eta_{j} \zeta_{j+1}^{*}$ for $j=1,2, \ldots, n-1$. We see from Eq. (55) that they are real linear combinations of $w_{1} \zeta_{1}^{*}, w_{2} \zeta_{2}^{*}, \ldots, w_{n-1} \zeta_{n-1}^{*}, w_{n} \zeta_{n}^{*}$,

$$
\eta_{j} \zeta_{j+1}^{*}=-\frac{\left|\zeta_{j+1}\right|^{2}}{\rho_{j} \rho_{j+1}} \sum_{k=1}^{j} \zeta_{j}^{*} w_{j}+\frac{\rho_{j}}{\rho_{j+1}} w_{j+1} \zeta_{j+1}^{*}
$$

Using both the orthogonality of $\underline{w}$ and $\zeta$, and the reduction process (51) for $\boldsymbol{\Delta}_{4}$ 's formed out of the last two columns of $A_{n}$, it is now clear that the set of complex invariants (47) can be replaced by the following set of $(n-2) \Delta_{4}$ 's:

$$
\boldsymbol{\Delta}_{j, n-1}=w_{j} . \zeta_{j}^{*} w_{j+1}^{*} \zeta_{j+1}, \quad j=1,2, \ldots, n-2 .
$$

The known algebraic independence of the set (47) implies a similar independence of these $\boldsymbol{\Delta}_{4}$ 's.

To tackle the next set of $(n-3)$ invariants $\xi_{j} \eta_{j+1}^{*} \xi_{j+1}^{*} \eta_{j+2}$ for $j=1,2, \ldots, n-3$ in the list (48), we must bring in the $(n-2)$ th column of the matrix $A_{n}$. Denote this by $\underline{v} \in \mathcal{B}_{n}$ so that

$$
A_{n}=\left(\begin{array}{cccccc}
\cdot & \cdot & \cdot & v_{1} & w_{1} & \zeta_{1} \\
\cdot & \cdot & \cdot & v_{2} & w_{2} & \zeta_{2} \\
\cdot & \cdot & \cdot & \vdots & \vdots & \vdots \\
\cdot & \cdot & \cdot & v_{n} & w_{n} & \zeta_{n}
\end{array}\right) .
$$

Analogous to Eq. (54) we now have

$$
A_{n-2}(\underline{\xi}) A_{n-3}=A_{n-1}(\underline{\eta})^{\dagger} A_{n}(\underline{\zeta})^{\dagger} A_{n},
$$

from where we get expressions for $\xi_{j}$ in terms of $\zeta$, $\eta$, and $\underline{\mathrm{v}}$. This is naturally more complicated than Eq. (55) at the previous stage. In place of the real positive factors $\rho_{j}$ defined in terms of $\zeta$ in Eq. (28), we now have similarly defined factors $\sigma_{j}$ in terms of $\eta$ occurring in the elements of $A_{n-1}(\eta)$. The result of comparing the $(n-2)$ th columns of both sides of Eq. (60) is

$$
\begin{aligned}
\xi_{j}= & \frac{\sigma_{j} \rho_{j+1}}{\sigma_{j+1} \rho_{j+2}} v_{j+2}-\frac{\sigma_{j}}{\sigma_{j+1} \rho_{j+1} \rho_{j+2}} \zeta_{j+2} \sum_{\ell=1}^{j+1} \zeta_{\ell}^{*} v_{\ell} \\
& -\frac{\eta_{j+1}}{\sigma_{j} \sigma_{j+1}} \sum_{k=1}^{j} \frac{\rho_{k}}{\rho_{k+1}} \eta_{k}^{*} v_{k+1} \\
& +\frac{\eta_{j+1}}{\sigma_{j} \sigma_{j+1}} \sum_{k=1}^{j} \frac{\eta_{k}^{*} \zeta_{k+1}}{\rho_{k} \rho_{k+1}} \sum_{\ell=1}^{k} \zeta_{\ell}^{*} v_{\ell}, \quad j=1,2, \ldots, n-2 .
\end{aligned}
$$

Here next we can use Eq. (55) to go from $\eta$ to $\underline{w}$. Then we form the expressions $\xi_{j} \eta_{j+1}^{*}$ and step by step work our way up to the invariants $\xi_{j} \eta_{j+1}^{*} \xi_{j+1}^{*} \eta_{j+2}$. We can then see that apart from various real factors we encounter $\boldsymbol{\Delta}_{4}$ 's involving $v$ 's, and $w$ 's, $v$ 's and ' 's, and $w$ 's and $\zeta$ 's. Using the reduction rules (51) the $v-\zeta$ combinations can be eliminated in favor of the other two types. It is now clear that apart from the $\boldsymbol{\Delta}_{j, n-1}$ in Eq. (58) that appeared at the previous stage, the new quantities that come in now are $\boldsymbol{\Delta}_{j, n-2}$. But we know in advance that at this stage only $(n-3)$ new independent invariants are available. As all the rows of $\boldsymbol{\Delta}_{n}$ are on equal footing, we conclude that the new $\boldsymbol{\Delta}_{4}$ 's to be added now to the previous $\boldsymbol{\Delta}_{j, n-1}$ may be taken to be

$$
\boldsymbol{\Delta}_{j, n-2}=v_{j} w_{j}^{*} v_{j+1}^{*} w_{j+1}, \quad j=1,2, \ldots,(n-3) .
$$

In this manner, one sees recursively that the $\frac{1}{2}(n-1)(n$ $-2)$ independent gauge-invariant phases in a general matrix $A_{n} \in \mathrm{U}(n)$ are the Bargmann invariants $\boldsymbol{\Delta}_{j k}$ for $j<k \leqslant n-1$. In any case such a choice is permitted. However, the actual algebraic expression of a general $\boldsymbol{\Delta}_{j k}$ in terms of this special subset may be rather involved, so one may freely use all $\boldsymbol{\Delta}_{j k}$ in constructing interesting gauge-invariant expressions with various properties.

The upshot of these considerations is that the naturally available gauge-invariant phases for the continuous unitary evolution of an $n$-level quantum system, barring degeneracies and level crossings, are $n$ geometric phases $\varphi_{g}\left[C_{j}\right]$ as defined in Eq. (13), and the $(n-1)^{2}$ primitive four-vertex Bargmann invariants $\boldsymbol{\Delta}_{j k}$ of Eq. (52); of the latter, only the $\frac{1}{2}(n-1)(n-2) \boldsymbol{\Delta}_{j k}$ 's for $j<k \leqslant n-1$ are independent. Any composite expression formed out of these ingredients is, of course, also invariant.

\section{OFF-DIAGONAL GEOMETRIC PHASES}

It is evident from the definitions (6) that while the dynamical phase $\varphi_{\text {dyn }}[\mathcal{C}]$ is always numerically well defined once the parametrized curve $\mathcal{C}$ is given, the total phase $\varphi_{\text {tot }}[\mathcal{C}]$ is only defined modulo $2 \pi$ and, moreover, is undefined if the vectors $\psi\left(s_{1}\right)$ and $\psi\left(s_{2}\right)$ at the end points of $\mathcal{C}$ are orthogonal. These properties naturally carry over to the geometric phase $\varphi_{g}[C]$ : only defined modulo $2 \pi$, undefined when $\varphi_{\text {tot }}[\mathcal{C}]$ is undefined. The Bargmann invariants (7) too share these problems of definition as far as their phases are concerned, which explains the limitation to generic situations.

Recently a very interesting attempt to define so-called offdiagonal geometric phases has been made to cover just these exceptional or problematic situations [11]. Specifically, the idea is to set up gauge-invariant phases associated with the unitary evolution of an $n$-level quantum system, which remain well defined even when one of the eigenvectors of the Hamiltonian at a final time $t_{2}$, say the $k$ th one, happens to coincide with the $j$ th eigenvector at the initial time $t_{1}$, with $j \neq k$. In this situation, as $\psi_{j}\left(t_{1}\right)$ and $\psi_{k}\left(t_{2}\right)$ are the same up to a phase, both the geometric phases $\varphi_{g}\left[C_{j}\right]$ and $\varphi_{g}\left[C_{k}\right]$ become undefined since the inner products $\left[\psi_{j}\left(t_{1}\right), \psi_{j}\left(t_{2}\right)\right]$ and $\left[\psi_{k}\left(t_{1}\right), \psi_{k}\left(t_{2}\right)\right]$ vanish. We shall now briefly recall the basic quantities introduced in this new approach and then show that the usual geometric phases and Bargmann invariants as defined earlier can completely handle the new situation. It is just that they must be put together in such combi- 
nations so that the potentially undefined factors in each precisely cancel one another in exceptional situations.

The notation for the evolution of an $n$-level quantum system is as given in Sec. III. The quantities defined in the off-diagonal geometric phases method, when expressed in our notations, are

$$
\begin{gathered}
I_{j}=\exp \left\{-i \varphi_{\text {dyn }}\left[\mathcal{C}_{j}\right]\right\}, \quad j=1,2, \ldots, n, \\
\sigma_{j k}=\exp \left\{i \arg \left(\psi_{j}, \phi_{k}\right)-i \varphi_{\text {dyn }}\left[\mathcal{C}_{k}\right]\right\}, \quad j \neq k, \\
\gamma_{j k}=\sigma_{j k} \sigma_{k j}, \quad j \neq k, \\
\gamma_{j}=\exp \left\{i \varphi_{g}\left[C_{j}\right]\right\}, \quad j=1,2, \ldots, n .
\end{gathered}
$$

Of these, $I_{j}$ and $\sigma_{j k}$ are not gauge invariant, but $\gamma_{j k}$ and $\gamma_{j}$ are gauge invariant. In case for some $j \neq k$ we have $\left|\left(\psi_{j}, \phi_{k}\right)\right|=1$, it is clear that both $\varphi_{g}\left[C_{j}\right]$ and $\varphi_{g}\left[C_{k}\right]$ become undefined, but the "off-diagonal" quantity $\gamma_{j k}$ remains well defined.

The two-state or two-index quantity $\gamma_{j k}$ has been generalized to a multi-index quantity of order $\ell$ as follows:

$$
\gamma_{j_{1} j_{2} \cdots j \ell}=\sigma_{j_{1} j_{2}} \sigma_{j_{2} j_{3}} \cdots \sigma_{j_{\ell-1} j_{\ell}} \sigma_{j_{\ell} j_{1}},
$$

and this again is gauge invariant.

We can now see that all these newly introduced gaugeinvariant off-diagonal quantities $\gamma_{j k}, \gamma_{j_{1} j_{2} \ldots j}$, are actually expressible completely in terms of the geometric phases and Bargmann invariants for the $n$-level system, in carefully chosen combinations,

$$
\begin{aligned}
\gamma_{j k}=\exp \left\{i \arg \Delta_{4}\left(\psi_{j}, \phi_{k}, \psi_{k}, \phi_{j}\right)+i \varphi_{g}\left[C_{j}\right]+i \varphi_{g}\left[C_{k}\right]\right\} \\
\gamma_{j_{1} j_{2} \ldots j \ell}=\exp \left\{i \arg \Delta_{2} \ell\left(\phi_{j_{1}}, \psi_{j_{1}}, \phi_{j_{2}}, \psi_{j_{2}}, \ldots, \phi_{j \ell}, \psi_{j_{\ell}}\right)\right. \\
\left.+i \varphi_{g}\left[C_{j_{1}}\right]+i \varphi_{g}\left[C_{j_{2}}\right]+\cdots+i \varphi_{g}\left[C_{j_{\ell}}\right]\right\}
\end{aligned}
$$

In the case of $\gamma_{j k}$, for example, we see that when $\left|\left(\psi_{j}, \phi_{k}\right)\right|=1$ and $\varphi_{g}\left[C_{j}\right], \varphi_{g}\left[C_{k}\right]$ become undefined because the total phases $\varphi_{\text {tot }}\left[\mathcal{C}_{j}\right]$ and $\varphi_{\text {tot }}\left[\mathcal{C}_{k}\right]$ are undefined, there are compensating factors from $\boldsymbol{\Delta}_{4}\left(\psi_{j}, \phi_{k}, \psi_{k}, \phi_{j}\right)$ that precisely cancel these parts of the individual geometric phases, so that $\gamma_{j k}$ remains unambiguous. The mechanism is similar in the case of the higher-order expressions $\gamma_{j_{1} j_{2}} \cdots j_{\ell}$.

It has been shown that $\gamma_{j_{1} j_{2}} \ldots j$, for $\ell \geqslant 4$ can be reduced to the expressions with $\ell=2$ and $\ell=3$, so these are the primitive ones. Among these, we can limit ourselves to choices obeying $j_{1}<j_{2}$ when $\ell=2$ and $j_{1}=1<j_{2}<j_{3}$ when $\ell=3$, in counting independent quantities. However, the upshot of our analysis is that we can always work with just the geometric phases $\varphi_{g}\left[C_{j}\right]$ and the independent $\boldsymbol{\Delta}_{4}$ 's listed in the previous section (but for convenience employ all the $\boldsymbol{\Delta}_{j k}$ if necessary). All gauge-invariant quantities can be built up out of them, so that conceptually the off-diagonal geometric phases are constructed out of previously known familiar building blocks.

\section{CONCLUDING REMARKS}

We have carried out a complete analysis of the gaugeinvariant objects for $n$-level quantum systems. This entails introduction of Bargmann invariants defined over two sets of orthonormal basis vectors, demonstration that the primitive Bargmann invariants are four-vertex Bargmann invariants, and finally the identification of an algebraically independent set of four-vertex Bargmann invariants that turn out to be $(n-1)(n-2) / 2$ in number. In the process of achieving this task we developed a canonical form for $\mathrm{U}(n)$ matrices in terms of a sequence of complex unit vectors of dimensions $n, n-1, \ldots, 1$ which may be useful in other contexts as well. Indeed, this form has already found application in parametrizing the CKM matrices that arise in the context of $C P$ violation in particle physics. The gauge-invariant building blocks constructed here are shown to provide a complete quantum kinematic picture of the recently discovered offdiagonal phases. The usefulness of the off-diagonal phases is thus extended far beyond the restrictive framework of adiabatic evolution. This reinforces the view that the Bargmann invariants and the traditional geometric phases, and suitably constructed combinations of them, suffice in answering all interesting questions in this domain.
[1] M.V. Berry, Proc. R. Soc. London, Ser. A 392, 45 (1984).

[2] N. Mukunda and R. Simon, Ann. Phys. (N.Y.) 228, 205 (1993); ibid. 228, 269 (1993).

[3] Y. Aharonov and J. Anandan, Phys. Rev. Lett. 58, 1593 (1987).

[4] J. Samuel and R. Bhandari, Phys. Rev. Lett. 60, 2339 (1988).

[5] Many of the early papers on geometric phase have been reprinted in Geometric Phases in Physics, edited by A. Shapere and F. Wilczek (World Scientific, Singapore, 1989); and in Fundamentals of Quantum Optics, SPIE Milestone Series, edited by G. S. Agarwal (SPIE Press, Bellington, 1995).

[6] E.M. Rabei, Arvind, N. Mukunda, and R. Simon, Phys. Rev. A 60, 3397 (1999).

[7] V. Bargmann, J. Math. Phys. 5, 862 (1964).
[8] W.R. Hamilton, Lectures on Quaternions (Dublin, 1853); L.C. Biedenharn and J.D. Louck, Angular Momentum in Quantum Physics, Encyclopedia of Mathematics and its applications, Vol. 8 (Addison-Wesley, Reading, MA, 1981). Hamilton's theory of turns has been generalized to the simplest noncompact semisimple group $\mathrm{SU}(1,1) \sim \mathrm{SL}(2, R) \sim \mathrm{Sp}(2, R)$ in R. Simon, N. Mukunda, and E.C.G. Sudarshan, Phys. Rev. Lett. 62, 1331 (1989); J. Math. Phys. 30, 1000 (1989). and to the Lorentz group in R. Simon, S. Chaturvedi, V. Srinivasan, and N. Mukunda, Hamilton's Turns for the Lorentz Group (The Institute of Mathematical Sciences, Chennai, in press); the relationship between Hamilton's turns and geometric phase for twolevel systems is described in R. Simon and N. Mukunda, J. Phys. A 25, 6135 (1992). 
[9] R. Simon and N. Mukunda, Phys. Rev. Lett. 70, 880 (1993).

[10] S. Chaturvedi and N. Mukunda, Int. J. Mod. Phys. A 16, 1481 (2001).

[11] F. Pistolesi and N. Manini, Phys. Rev. Lett. 85, 1585 (2000); N. Manini and F. Pistolesi, ibid. 85, 3067 (2000).

[12] D.E. Manolpoulos and M.S. Child, Phys. Rev. Lett. 82, 2223 (1999).

[13] S. Panchratnam, Proc.-Indian Acad. Sci., Sect. A 44, 247
(1956); S. Ramaseshan and R. Nityananda, Curr. Sci. 55, 1225 (1986).

[14] D. Page, Phys. Rev. A 36, 3479 (1987).

[15] S. Kobayashi and K. Nomizu, Foundations of Differential Geometry (Interscience Publishers, New York, 1969), Vol. II, Chap. 9.

[16] H. Harari and M. Leurer, Phys. Lett. B 181, 123 (1986); J.F. Nieves and P.B. Pal, Phys. Rev. D 36, 315 (1987). 